\title{
NEW DENSE SUPERBALL PACKINGS IN THREE DIMENSIONS
}

\author{
MARIA DOSTERT AND FRANK VALLENTIN
}

\begin{abstract}
In this paper we construct a new family of lattice packings for superballs in three dimensions (unit balls for the $l_{3}^{p}$ norm) with $p \in(1,1.58]$. We conjecture that the family also exists for $p \in\left(1.58, \log _{2} 3=1.5849625 \ldots\right]$. Like in the densest lattice packing of regular octahedra, each superball in our family of lattice packings has 14 neighbors.
\end{abstract}

\section{INTRODUCTION}

Finding dense packings of spheres in $n$-dimensional Euclidean space is one of the most central problems in discrete geometry. In this paper, we consider lattice packings of superballs in dimension three. Superballs are unit balls for the $\ell_{n}^{p}$ norm:

$$
\mathcal{B}_{n}^{p}=\left\{x \in \mathbb{R}^{n}:\|x\|_{p} \leq 1\right\} \text { where }\|x\|_{p}=\left(\sum_{i=1}^{n}\left|x_{i}\right|^{p}\right)^{1 / p} .
$$

We distinguish between three different kinds of packings which are increasingly restrictive: congruent packings, translative packings, and lattice packings. Let $\mathcal{K}$ be a convex body in $\mathbb{R}^{n}$. By $\mathcal{K}^{\circ}$ we denote its topological interior. We consider the special orthogonal group

$$
\mathrm{SO}(n)=\left\{A \in \mathbb{R}^{n \times n}: A A^{\top}=I_{n}, \operatorname{det} A=1\right\},
$$

and $I_{n}$ denotes the identity matrix.

A congruent packing of $\mathcal{K}$ is defined as

$$
\mathcal{P}=\bigcup_{i \in \mathbb{N}}\left(x_{i}+A_{i} \mathcal{K}\right) \text { with }\left(x_{i}, A_{i}\right) \in \mathbb{R}^{n} \times \mathrm{SO}(n),
$$

where

$$
\left(x_{i}+A_{i} \mathcal{K}^{\circ}\right) \cap\left(x_{j}+A_{j} \mathcal{K}^{\circ}\right)=\emptyset \text { whenever } i \neq j .
$$

Translative packings are congruent packings without rotations, i.e. all matrices $A_{i}$ are equal to $I_{n}$. Moreover, if the set $\left\{x_{i}: i \in \mathbb{N}\right\}$ forms a lattice (a discrete subgroup of $\left.\mathbb{R}^{n}\right)$, then we call this packing a lattice packing.

The (upper) density of a congruent packing $\mathcal{P}$ is defined as

$$
\delta(\mathcal{P})=\limsup _{r \rightarrow \infty} \sup _{c \in \mathbb{R}^{n}} \frac{\operatorname{vol}(B(c, r) \cap \mathcal{P})}{\operatorname{vol} B(c, r)},
$$

where $B(c, r)=\left\{x \in \mathbb{R}^{n}:\|x-c\|_{2} \leq r\right\}$ is the Euclidean ball of radius $r$ with center $c$.

Date: June 27, 2018.

1991 Mathematics Subject Classification. 11H31, 52C17, 90C30.

Key words and phrases. lattice packings, superballs, interval arithmetic. 
Jiao, Stillinger, and Torquato [11, 12] determined four families of dense superball packings via computer simulations. They divided the possible values of $p$ into four regimes:

$$
p \in[1, \infty)=\left[1, \log _{2} 3=1.5849 \ldots\right] \cup\left[\log _{2} 3,2\right] \cup[2,2.3018 \ldots] \cup[2.3018 \ldots, \infty) .
$$

For every regime they found a family of lattices which defines dense lattice packings for $\mathcal{B}_{3}^{p}$. The family for the first regime $\left[1, \log _{2} 3\right]$ they call the $\mathbb{O}_{1}$ lattices, and $\mathbb{O}_{0}, \mathbb{C}_{0}$, $\mathbb{C}_{1}$ correspondingly for the second, third, and fourth regime. In their approach, they even allowed for congruent packings, but it turned out that the densest congruent packings they obtained were lattice packings. They conjectured that they found the densest congruent packings of $\mathcal{B}_{3}^{p}$ for all values of $p$.

However, Ni, Gantapara, de Graaf, van Roij, and Dijkstra 14 found, also via computer simulations, denser lattice packings for superballs in the second regime. Also for $p=1.4$, which falls into the first regime, they give one lattice for which they claim (but see Section 3 ) that it is denser than the corresponding $\mathbb{O}_{1}$ lattice. They report (see page 8830 in [14]) that due to numerical instabilities they could not investigate values $p<1.4$.

In this paper, we use a method of Minkowski [13] to determine locally densest lattice packings of $\mathcal{B}_{3}^{p}$. Minkowski applied this method to determine the densest lattice packing of regular octahedra $\mathcal{B}_{3}^{1}$. Starting from Minkowski's lattice we found a family of new lattice packings for the first regime which is denser than the $\mathbb{O}_{1}$ lattices, see Table 1. Here, each superball in our family of lattice packings has 14 neighbors, like in the densest lattice packing of regular octahedra. We also found new lattice packings in the second regime which are denser than the $\mathbb{O}_{0}$ lattices, see Table 2. For the third and fourth regime the densest lattices we found are the $\mathbb{C}_{0}$ lattices, respectively the $\mathbb{C}_{1}$ lattices.

$\begin{array}{ccc}p & \text { packing density of } \mathbb{O}_{1} \text { lattices } & \text { packing density of new family } \\ 1 & 18 / 19=0.94736 \ldots & 0.94736 \ldots \\ 1.1 & 0.90461 \ldots & 0.90913 \ldots \\ 1.2 & 0.87121 \ldots & 0.87861 \ldots \\ 1.3 & 0.84516 \ldots & 0.85375 \ldots \\ 1.4 & 0.82497 \ldots & 0.83284 \ldots \\ 1.5 & 0.80948 \ldots & 0.81395 \ldots \\ \log _{2} 3 & 0.79594 \ldots & 0.79594 \ldots\end{array}$

TABLE 1. Packing density of $\mathbb{O}_{1}$ lattices (see [11, 12] ) and of our new family (see Section 3 and Section 4). When $p=1$ we obtained the lattice which determines the densest lattice packing of regular octahedra (see [13]) and when $p=\log _{2} 3$ we obtain the body centered cubic lattice.

The structure of the paper is as follows: We first explain Minkowski's method and how we approached it computationally in Section 2 Section 3 contains a report on some of our findings, in particular new lattice packings in the first and second regime. Section 4 provides a computer-assisted proof proving that the family of new dense lattice packings exists for $p \in[1,1.58]$. We end with Section 5 by posing some conjectures and open problems. 


$\begin{array}{ccc}p & \text { packing density of } \mathbb{O}_{0} \text { lattices } & \text { packing density of new lattices } \\ \log _{2} 3 & 0.79594 \ldots & 0.79594 \ldots \\ 1.6 & 0.79084 \ldots & 0.79084 \ldots \\ 1.7 & 0.76567 \ldots & 0.76610 \ldots \\ 1.8 & 0.75126 \ldots & 0.75303 \ldots \\ 1.9 & 0.74364 \ldots & 0.74550 \ldots \\ 2 & \pi / \sqrt{18}=0.74048 \ldots & 0.74048 \ldots\end{array}$

TABle 2. Packing density of $\mathbb{O}_{0}$ lattices (see [1, 12, ) and of the new lattices (see [14] and see Section 3). When $p=2$ we obtain the face centered cubic lattice.

\section{Determination of LAtTiCe PACKings By LOCAL OPtimization}

Let $\Lambda$ be a lattice so that

$$
\mathcal{P}=\bigcup_{x \in \Lambda}(x+\mathcal{K})
$$

forms a lattice packing of the convex body $\mathcal{K}$, i.e. for all distinct lattice vectors $x, y \in \Lambda$ we have

$$
\left(x+\mathcal{K}^{\circ}\right) \cap\left(y+\mathcal{K}^{\circ}\right)=\emptyset .
$$

Then, $\Lambda$ is called a packing lattice. From (1) it immediately follows that a packing lattice is characterized by

$$
\Lambda \cap(\mathcal{K}-\mathcal{K})^{\circ}=\emptyset, \text { where } \mathcal{K}-\mathcal{K}=\{x-y: x, y \in \mathcal{K}\}
$$

is the difference body of $\mathcal{K}$. In other words, $\Lambda$ is a packing lattice for $\mathcal{K}$ if and only if the condition

$$
\|x\|_{\mathcal{K}-\mathcal{K}} \geq 1 \text { for all } x \in \Lambda \backslash\{0\}
$$

holds; here

$$
\|x\|_{\mathcal{L}}=\inf \left\{\lambda: \frac{1}{\lambda} x \in \mathcal{L}\right\}
$$

is the Minkowski norm of $x$ defined by a centrally symmetric convex body $\mathcal{L}$.

The general linear group of degree $n$ over a ring $R$ is defined as

$$
\mathrm{GL}_{n}(R)=\left\{B \in R^{n \times n}: \exists A \in R^{n \times n}: A B=B A=I_{n}\right\} .
$$

A matrix $B \in \mathrm{GL}_{n}(\mathbb{R})$ with linearly independent column vectors $b_{1}, \ldots, b_{n}$ specifies a lattice $\Lambda$ by taking all integral linear combinations of $b_{1}, \ldots, b_{n}$ :

$$
\Lambda=\mathbb{Z} b_{1}+\cdots+\mathbb{Z} b_{n}=B \mathbb{Z}^{n} .
$$

Two matrices $B, B^{\prime} \in \mathrm{GL}_{n}(\mathbb{R})$ determine the same lattice if and only if there is a matrix $U \in \mathrm{GL}_{n}(\mathbb{Z})$ such that $B U=B^{\prime}$. Matrix $B$ also gives a fundamental domain $F$ of $\Lambda$ by

$$
F=\left\{\sum_{i=1}^{n} \alpha_{i} b_{i}: \alpha_{i} \in[0,1], i=1, \ldots, n\right\}
$$


Then, the volume of a fundamental domain of $\Lambda$ is $\operatorname{vol}\left(\mathbb{R}^{n} / \Lambda\right)=\operatorname{vol} F=|\operatorname{det}(B)|$. The density of a lattice packing $\mathcal{P}=\bigcup_{x \in \Lambda}(x+\mathcal{K})$ is

$$
\delta(\mathcal{P})=\frac{\operatorname{vol} \mathcal{K}}{\operatorname{vol}\left(\mathbb{R}^{n} / \Lambda\right)}=\frac{\operatorname{vol} \mathcal{K}}{|\operatorname{det} B|} .
$$

So one can state the problem of finding a densest lattice packing of a convex body $\mathcal{K}$ as the following minimization problem:

$$
\begin{aligned}
\operatorname{minimize} & |\operatorname{det} B| \\
\text { so that } & B \in \mathrm{GL}_{3}(\mathbb{R}) / \mathrm{GL}_{3}(\mathbb{Z}) \\
& \|B u\|_{\mathcal{K}-\mathcal{K}} \geq 1 \text { for all } u \in \mathbb{Z}^{n} \backslash\{0\} .
\end{aligned}
$$

It follows from Mahler's selection theorem that the minimum is attained, see [6, Theorem 30.1].

Two distinct translates $x+\mathcal{K}$ and $y+\mathcal{K}$ are called neighbors in a lattice packing $\mathcal{P}$ if they have a nonempty intersection. The number of neighbors coincides for all translates. How many neighbors can $\mathcal{K}$ have at most? Minkowski showed that $\mathcal{K}$ has at most $3^{n}-1$ neighbors and if $\mathcal{K}$ is strictly convex, then the number of neighbors is bounded by $2^{n+1}-2$, see [6, Theorem 30.2]. Swinnerton-Dyer proved that when a lattice $\Lambda$ achieves a density which is locally maximal, then $\mathcal{K}$ has at least $n(n+1)$ neighbors, see [6, Theorem 30.3].

Let $B \in \mathrm{GL}_{3}(\mathbb{R})$ be a matrix defining a locally densest lattice packing $\Lambda=B \mathbb{Z}^{3}$ of $\mathcal{K}$. Minkowski [13] (see also [7, §32], [1, [9]) showed that after performing a suitable $\mathrm{GL}_{n}(\mathbb{Z})$-transformation to $B$ we can reduce to the following three cases.

Case (I): $B u+\mathcal{K}$ with $u \in \mathcal{U}^{1}$ are neighbors of $\mathcal{K}$, where

$$
\mathcal{U}^{1}=\{ \pm(1,0,0), \pm(0,1,0), \pm(0,0,1), \pm(1,-1,0), \pm(0,1,-1), \pm(1,0,-1)\},
$$

but $\pm(-1,1,1), \pm(1,-1,1), \pm(1,1,-1)$ are not.

Case (II): $B u+\mathcal{K}$, with $u \in \mathcal{U}^{2}$, are neighbors of $\mathcal{K}$, where

$$
\mathcal{U}^{2}=\{ \pm(1,0,0), \pm(0,1,0), \pm(0,0,1), \pm(1,1,0), \pm(0,1,1), \pm(1,0,1)\},
$$

but $\pm(1,1,1)$ are not.

Case (III): $B u+\mathcal{K}$, with $u \in \mathcal{U}^{3}$, are neighbors of $\mathcal{K}$, where

$$
\mathcal{U}^{3}=\mathcal{U}^{2} \cup\{ \pm(1,1,1)\} .
$$
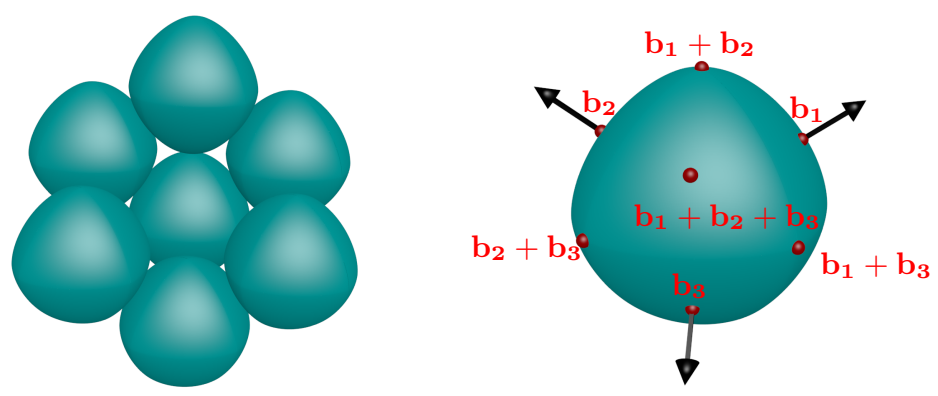

Figure 1. On the left: A part of a lattice packing for $B_{3}^{\log _{2} 3}$ satisfying Case (III). On the right: Seven of fourteen neighbors of the packing. Contact points are labeled in red. 
From now on, we are interested in lattice packings of superballs in three dimensions. We perform a rescaling by setting $\mathcal{K}=\frac{1}{2} \mathcal{B}_{3}^{p}$ so that $\|x\|_{\frac{1}{2} \mathcal{B}_{3}^{p}-\frac{1}{2} \mathcal{B}_{3}^{p}}=\|x\|_{p}$. Note that scaling $\mathcal{K}$ only scales the packing lattices but does not effect the packing density.

For each of the three cases, Case (I), (II), and (III), we can numerically find critical points of the following finite nonlinear optimization problem

$$
\begin{aligned}
& \text { minimize } \operatorname{det} B \\
& \text { so that } B \in \mathrm{GL}_{3}(\mathbb{R}) \\
& \qquad \operatorname{det} B>0 \\
& \|B u\|_{p}=1 \text { for all } u \in \mathcal{U}^{i},
\end{aligned}
$$

for $i=1,2,3$, in order to identify candidates for locally densest lattice packings.

After finding a matrix $B$ which is a feasible solution of this optimization problem, we have to check whether $B$ indeed defines a packing lattice for $\mathcal{B}_{3}^{p}$. For this it suffices to verify that $\|B u\|_{p} \geq 1$ holds for only finitely many vectors $u \in \mathbb{Z}^{n} \backslash\{0\}$ as the following lemma shows; see also Dieter [3].

Lemma 2.1. Suppose $p, q \in[1, \infty)$ satisfy the relation $\frac{1}{p}+\frac{1}{q}=1$ and suppose that a matrix $B \in \mathrm{GL}_{n}(\mathbb{R})$ is given. If $u=\left(u_{1}, \ldots, u_{n}\right) \in \mathbb{Z}^{n}$ is such that $\|B u\|_{p} \leq \mu$ for some nonnegative number $\mu$, then

$$
\left|u_{i}\right| \leq\left\|B_{i}^{-1}\right\|_{q} \mu \quad \text { for all } i=1, \ldots, n,
$$

where $B_{i}^{-1}$ is the $i$-th row vector of $B^{-1}$.

Proof. We apply the triangle inequality and Hölder's inequality, and get

$$
\left|u_{i}\right|=\left|B_{i}^{-1} B u\right| \leq \sum_{j=1}^{n}\left|B_{i, j}^{-1}(B u)_{j}\right| \leq\left\|B_{i}^{-1}\right\|_{q}\|B u\|_{p} \leq\left\|B_{i}^{-1}\right\|_{q} \mu .
$$

If $1<p<2$ and if $B \in \mathrm{GL}_{3}(\mathbb{R})$ satisfies the equality conditions of Case (III)

$$
\|B u\|_{p}=1 \text { for all } u \in \mathcal{U}^{3},
$$

then this extra check is not necessary, as the next lemma shows. This is a consequence of Hanner's inequality [8]

$$
\|x+y\|_{p}^{p}+\|x-y\|_{p}^{p} \geq\left(\|x\|_{p}+\|y\|_{p}\right)^{p}+\left|\|x\|_{p}-\|y\|_{p}\right|^{p},
$$

which holds for all $1<p<2$ and all $x, y \in \mathbb{R}^{n}$.

Lemma 2.2. If $1<p<2$ and if $B \in \mathrm{GL}_{3}(\mathbb{R})$ satisfies $\|B u\|_{p}=1$ for all $u \in \mathcal{U}^{3}$, then $\|B u\|_{p} \geq 1$ for all $u \in \mathbb{Z}^{n} \backslash\{0\}$.

Proof. As a start we consider $u=(1,-1,0)$. We write $u$ as the sum of two vectors $v, w$ in $\mathcal{U}^{3}: u=(1,0,0)+(0,-1,0)$. Then by Hanner's inequality (3) we get

$$
\begin{aligned}
\|B u\|_{p}^{p} & =\|B v+B w\|_{p}^{p} \\
& \geq\left(\|B v\|_{p}+\|B w\|_{p}\right)^{p}+\left|\|B v\|_{p}-\|B w\|_{p}\right|^{p}-\|B v-B w\|_{p}^{p} \\
& \geq 2^{p}-1 \geq 1 .
\end{aligned}
$$

The vectors $u=(1,0,-1),(0,1,-1)$ can be treated similarly.

Now we consider $u=(-1,1,1)$. We write $u$ as $u=v+w$ with $u=(0,1,1)$, $w=(-1,0,0)$ and apply Hanner's inequality to get $\|\left. B u\right|_{p} ^{p} \geq 2^{p}-1$. Again, the vectors $u=(1,-1,1),(1,1,-1)$ can be treated similarly. 
So we have $\|B u\|_{p} \geq 1$ for all $u \in \mathbb{Z}^{3} \backslash\{0\}$ with $\left|u_{i}\right| \leq 1$ and we proceed by induction. For the inductive step assume that $\|B u\|_{p} \geq 1$ for all $u \in \mathbb{Z}^{3} \backslash\{0\}$ with $\left|u_{i}\right| \leq M$. Let $u \in \mathbb{Z}^{3}$ with $\left|u_{i}\right| \leq M+1$ for all $i$ and $\left|u_{i}\right|=M+1$ for some $i$. Define $v, w \in \mathbb{Z}^{3}$ componentwise by

$$
v_{i}=\left\lceil\frac{u_{i}}{2}\right\rceil \text { and } w_{i}=\left\lfloor\frac{u_{i}}{2}\right\rfloor .
$$

Then, $\left|v_{i}\right|,\left|w_{i}\right| \leq M$. Hence, by the induction hypothesis, $B v \|_{p} \geq 1$, and $\|B w\|_{p} \geq$ 1. Since $v_{i}-w_{i} \in\{0,1\}$, we have $\|B v-B w\|_{p}=1$. Hanner's inequality (3) implies the desired inequality $\|B u\|_{p}^{p} \geq 2^{p}-1 \geq 1$.

\section{NUMERICAL FINDINGS}

In order to explore dense lattice packings of superballs in three dimensions we numerically found critical points of the nonlinear minimization problem (2). We randomly chose a matrix $B \in \mathrm{GL}_{3}(\mathbb{R})$, where we chose the matrix entries randomly according to the normal distribution $N(0,1)$ with mean 0 and variance 1 . Then we applied Newton's method to $B$ to find a critical point in the neighborhood of $B$. By applying this procedure to 10,000 randomly chosen starting points, we obtained a set of packing lattices. For the implementation we used the function root of the python package scipy.optimize, see [10]. We determined feasible packing lattices for each of these three cases.

3.1. First regime. The highest packing densities over all obtained solutions we found for $p=1,1.1,1.2,1.3,1.4,1.5$ belong to Case (III). We list them in Table 3 and we analyze them in detail in Section 4. The density we obtained for $p=1.4$ coincides with the value reported in [14, but the basis $e_{1}, e_{2}, e_{3}$ given in [14, page 8829] does not give a packing lattice since the $\ell_{3}^{p}$-norm of $2 e_{1}-e_{2}-e_{3}$ is too small.

$$
\begin{aligned}
L_{1} & =\left(\begin{array}{ccc}
-0.333333333333 & 0.166666666667 & 0.5 \\
0.5 & -0.3333333333333 & 0.1666666666667 \\
0.166666666667 & 0.5 & -0.333333333333
\end{array}\right) \\
L_{1.1} & =\left(\begin{array}{ccc}
-0.364125450067 & 0.193419513868 & 0.539049770666 \\
0.539049770666 & -0.364125450067 & 0.193419513867 \\
0.193419513867 & 0.539049770666 & -0.364125450068
\end{array}\right) \\
L_{1.2} & =\left(\begin{array}{ccc}
-0.392613644302 & 0.22381214158 & 0.569113821114 \\
0.569113821115 & -0.392613644298 & 0.223812141583 \\
0.223812141575 & 0.569113821114 & -0.392613644306
\end{array}\right) \\
L_{1.3} & =\left(\begin{array}{ccc}
-0.419839537546 & 0.260336714788 & 0.589023079183 \\
0.589023079194 & -0.419839537534 & 0.260336714788 \\
0.260336714754 & 0.589023079183 & -0.419839537578
\end{array}\right) \\
L_{1.4} & =\left(\begin{array}{ccc}
-0.4469847768893 & 0.307534456657 & 0.595696355817 \\
0.595696355844 & -0.446984776872 & 0.307534456649 \\
0.307534456588 & 0.595696355814 & -0.446984776962
\end{array}\right) \\
L_{1.5} & =\left(\begin{array}{ccc}
-0.475292821919 & 0.375983627555 & 0.580059051165 \\
0.580059051205 & -0.475292821888 & 0.375983627545 \\
0.375983627482 & 0.58005905116 & -0.475292821997
\end{array}\right)
\end{aligned}
$$

TABLE 3. Matrices $L_{p} \in \mathrm{GL}_{3}(\mathbb{R})$ giving the densest lattice packing of $\mathcal{B}_{n}^{p}$ we found. They all belong to Case (III). 
3.2. Second regime. The highest packing density over all obtained solutions we found for $p=1.6,1.7,1.8,1.9,2.0$ belong to Case (I). We list them in Table 4. We were not able to identify a pattern. The density we obained for $p=1.7$ coincides with the value reported in [14, but in [14] a corresponding matrix is not given. For more computational results we refer to the thesis [4] of the first author.

$$
\begin{aligned}
L_{1.6}= & \left(\begin{array}{ccc}
-0.000274732684343 & 0.00144215026174 & -0.999980951403 \\
0.509783945989 & 0.500572697171 & -0.499408517681 \\
0.509509213365 & -0.499408255119 & -0.500850623006
\end{array}\right) \\
L_{1.7} & =\left(\begin{array}{ccc}
0.458033772615 & 0.556735224273 & 0.553047497039 \\
-0.530691863753 & 0.577007354869 & 0.459643833129 \\
-0.0936769789857 & -0.0202829019484 & 0.988672468644
\end{array}\right) \\
L_{1.8} & =\left(\begin{array}{ccc}
-0.330208442415 & -0.696395141028 & 0.551458413193 \\
0.624661955256 & -0.637870063365 & 0.316559795406 \\
0.237379400053 & -0.0588146621535 & 0.954027742247
\end{array}\right) \\
L_{1.9} & =\left(\begin{array}{ccc}
-0.325366212309 & -0.0828873750566 & 0.930867632285 \\
0.230698700286 & 0.676231149106 & 0.66666839749 \\
-0.697856599406 & 0.59207566084 & 0.335768664213
\end{array}\right) \\
L_{2} & =\left(\begin{array}{lll}
0.000000000000 & 0.707106781187 & 0.707106781187 \\
0.707106781623 & 0.00000000000 & 0.70710678075 \\
0.707106781623 & 0.70710678075 & 0.000000000000
\end{array}\right)
\end{aligned}
$$

TABLE 4. Matrices $B_{p} \in \mathrm{GL}_{3}(\mathbb{R})$ giving the densest lattice packing of $\mathcal{B}_{n}^{p}$ we found. They all belong to Case (I). $L_{2}$ determines the face centered cubic lattice which defines a densest sphere packing in three dimensions.

\section{A new family of Lattice PaCkings}

When looking at the numerical solutions we found in the first regime, one immediately comes to the conclusion that the new found lattices belong to a family of lattices which one can easily parametrize.

Consider

$$
L(x, y, z) \in \mathrm{GL}_{3}(\mathbb{R}) \quad \text { with } \quad L(x, y, z)=\left(\begin{array}{ccc}
-x & y & z \\
z & -x & y \\
y & z & -x
\end{array}\right),
$$

where $x, y, z$ are chosen so that

$$
\|L(x, y, z) u\|_{p}=1 \text { for all } u \in \mathcal{U}^{3} .
$$

This implies

$$
\begin{aligned}
& \|L(x, y, z)(1,0,0)\|_{p}^{p}=|-x|^{p}+|y|^{p}+|z|^{p}=1 \\
& \|L(x, y, z)(1,1,0)\|_{p}^{p}=|-x+y|^{p}+|z-x|^{p}+|y+z|^{p}=1 \\
& \|L(x, y, z)(1,1,1)\|_{p}^{p}=3|-x+y+z|^{p}=1 .
\end{aligned}
$$

We also have the inequalities

$$
z \geq x \geq y \geq 0
$$


Thus, $x, y, z$ has to satisfy the following nonlinear system:

$$
\begin{aligned}
& z \geq x \geq y \geq 0 \\
& x^{p}+y^{p}+z^{p}=1 \\
& (x-y)^{p}+(z-x)^{p}+(y+z)^{p}=1 \\
& 3(-x+y+z)^{p}=1
\end{aligned}
$$

The family of lattices starts at $p=1$ with $x=1 / 3, y=1 / 6, z=1 / 2$, which defines the densest lattice packing of regular octahedra found by Minkowski. The family ends at $p=\log _{2} 3=1.5849625 \ldots$ with $x=y=z=\frac{1}{2}$ which defines the body centered cubic lattice.

We want to prove that the family indeed exists. When the nonlinear system (4) has a solution, Lemma 2.2 ensures that the members of the family are packing lattices for the corresponding superball $\frac{1}{2} B_{3}^{p}$. We will apply the following theorem of Cohn, Kumar, and Minton 2, Theorem 3.1], which is an effective implicit function theorem.

Theorem 4.1. Let $V$ and $W$ be finite-dimensional normed vector spaces over $\mathbb{R}$, and suppose that $f: B\left(x_{0}, \epsilon\right) \rightarrow W$ is a $C^{1}$ function, where $x_{0} \in V$ and $\varepsilon>0$. Suppose also that $T: W \rightarrow V$ is a linear operator such that

$$
\left\|D f(x) \circ T-i d_{W}\right\|<1-\frac{\|T\| \cdot\left|f\left(x_{0}\right)\right|}{\varepsilon}
$$

for all $x \in B\left(x_{0}, \varepsilon\right)$. Then there exists $x_{*} \in B\left(x_{0}, \varepsilon\right)$ such that $f\left(x_{*}\right)=0$. Moreover, in $B\left(x_{0}, \varepsilon\right)$, the zero locus $f^{-1}(0)$ is a $C^{1}$ submanifold of dimension $\operatorname{dim} V-\operatorname{dim} W$.

In the theorem, $\|\cdot\|$ denotes the operator norm, $D f(x)$ is the Jacobian of $f$ at $x$, and $i d_{W}$ is the identity operator on $W$, and $B\left(x_{0}, \varepsilon\right) \subseteq V$ is the open ball with center $x_{0}$ and radius $\varepsilon$, where the distance is measured using the norm of $V$.

Theorem 4.2. The nonlinear system (4) has a unique solution $\left(x_{*}(p), y_{*}(p), z_{*}(p)\right)$ for all $p \in[1,1.58]$. In particular, the matrix $L\left(x_{*}(p), y_{*}(p), z_{*}(p)\right)$ defines a packing lattice for $\frac{1}{2} \mathcal{B}_{3}^{p}$.

Proof. Our proof is computer assisted. We use interval arithmetic as implemented in the free open source mathematics software system SageMath [15].

Define the function $f_{p}: B\left(\left(x_{0}, y_{0}, z_{0}\right), \varepsilon\right) \rightarrow \mathbb{R}^{3}$ by

$$
f_{p}(x, y, z)=\left(\begin{array}{c}
x^{p}+y^{p}+z^{p}-1 \\
(x-y)^{p}+(z-x)^{p}+(y+z)^{p}-1 \\
3(-x+y+z)^{p}-1
\end{array}\right) .
$$

The Jacobian $D f_{p}(x, y, z)$ of $f_{p}$ equals

$$
p\left(\begin{array}{ccc}
x^{p-1} & y^{p-1} & z^{p-1} \\
(x-y)^{p-1}-(z-x)^{p-1} & -(x-y)^{p-1}+(y+z)^{p-1} & (z-x)^{p-1}+(y+z)^{p-1} \\
-3(-x+y+z)^{p-1} & 3(-x+y+z)^{p-1} & 3(-x+y+z)^{p-1}
\end{array}\right) .
$$

As norms we choose the $\ell_{3}^{\infty}$ norm for the domain, as well as for the codomain. Then the $\ell_{3}^{\infty}$ operator norm is the maximum of the $\ell_{3}^{1}$ norms of the rows of the considered matrix.

Now we subdivide the interval [1.1.58] in smaller subintervals and for every subinterval we choose an $\varepsilon>0$ and a starting point $\left(x_{0}, y_{0}, z_{0}\right)$ which we found by the numerical solution of the nonlinear system (4). 
Our choice of $\varepsilon>0$ and using the $\ell_{3}^{\infty}$ norm ensures that the ball $B\left(\left(x_{0}, y_{0}, z_{0}\right), \varepsilon\right)$ lies in the region $z \geq x \geq y \geq 0$. We set $T=D f_{p}\left(x_{0}, y_{0}, z_{0}\right)^{-1}$ and verify that inequality (5) is satisfied for all $p$ in the subinterval and for all $(x, y, z) \in$ $B\left(\left(x_{0}, y_{0}, z_{0}\right), \varepsilon\right)$. Then, the assumptions of Theorem 4.1 are satisfied and we can conclude that the nonlinear system (4) has a unique solution $\left(x_{*}(p), y_{*}(p), z_{*}(p)\right)$ for all $p$ in the subinterval. The verification of (5) uses interval arithmetic. Our Sage function verify is only a few lines long and can be found in Appendix A of this paper.

For example, we choose the subinterval $[1,1.01]$, the starting point $\left(x_{0}, y_{0}, z_{0}\right)=$ $\left(\frac{1}{3}, \frac{1}{6}, \frac{1}{2}\right)$, and $\varepsilon=0.03$. For $T$ we choose

$$
T=\left(\begin{array}{ccc}
\frac{1}{2} & 0 & -\frac{1}{6} \\
\frac{1}{2} & -\frac{1}{2} & \frac{1}{6} \\
0 & \frac{1}{2} & 0
\end{array}\right) .
$$

Then, Sage computes

$$
\left\|D f_{p}(x, y, z) T-I_{3}\right\| \in[0.00000000000000000,0.035585437892437462]
$$

and

$$
1-\frac{\|T\| \cdot\left|f_{p}\left(x_{0}, y_{0}, z_{0}\right)\right|}{\varepsilon} \in[0.60895579575438163,1.0000000000000000]
$$

for all $(x, y, z) \in B\left(\left(x_{0}, y_{0}, z_{0}\right), \varepsilon\right)$ and for all $p \in[1,1.01]$. This shows that (4) has a unique solution for each $p \in[1,1.01]$.

More examples of our choises can be found in Appendix B all choices can be found as ancillary file from the arXiv. org e-print archive.

We conjecture that the family of lattices also exists for $p \in\left(1.58, \log _{2} 3=\right.$ $1.5849625007 \ldots]$. We could enlarge the interval for which Theorem 4.2 holds by increasing the precision and by using smaller subintervals. For example, running the following Sage code shows that the lattice exists for $p \in[1.5849625,1.5849625+$ $\left.10^{-10}\right]$.

verify $(1.5849625,0.499999999842,0.499999124646,0.500000875038$, $0.0000002,0.0000000001)$

However, when $p$ approaches $\log _{2} 3$, then the Jacobian approaches

$$
D f_{\log _{2} 3}\left(\frac{1}{2}, \frac{1}{2}, \frac{1}{2}\right)=\log _{3} 2\left(\begin{array}{ccc}
\frac{2}{3} & \frac{2}{3} & \frac{2}{3} \\
0 & 1 & 1 \\
-2 & 2 & 2
\end{array}\right)
$$

which is singular. Furthermore, there is no $\varepsilon>0$ so that the ball $B\left(\left(\frac{1}{2}, \frac{1}{2}, \frac{1}{2}\right), \varepsilon\right)$ is contained in the region $z \geq x \geq y$. Currently, we do not know how to modify the approach to be able to handle these two difficulties.

\section{Conjectures and open problems}

Based on Section 3 we pose the following conjectures and open problems:

(1) The family of lattices determined by (4) exists for all $p \in\left(1.58, \log _{2} 3\right]$.

(2) The lattices we found in the first regime, and the ones found by Jiao, Stillinger, and Torquato [11] in the third and fourth regime give the densest lattice packings of superballs for the corresponding $p$.

(3) It would be interesting to develop a better understanding of the densest known lattices in the second regime. 
(4) Is there a value of $p \neq \infty$ for which the upper bound for translative packings of superballs determined in [5] matches the corresponding lower bound?

(5) For $p>\log _{2} 3$ there are no lattices which fall into Case (II) or into Case (III).

(6) What is the largest value of $p \leq 2$ so that the kissing number of $\mathcal{B}_{3}^{p}$ superballs is strictly larger than 12 ?

\section{ACKNOWLEDGEMENTS}

We thank Cristóbal Guzmán and Philippe Moustrou for helpful discussions.

This material is based upon work supported by the National Science Foundation under Grant No. DMS-1439786 while the first author was in residence at the Institute for Computational and Experimental Research in Mathematics in Providence, RI, during the "Point Configurations in Geometry, Physics and Computer Science" semester program. The second author was partially supported by the SFB/TRR 191 "Symplectic Structures in Geometry, Algebra and Dynamics", funded by the DFG. This project has received funding from the European Union's Horizon 2020 research and innovation programme under the Marie Skłodowska-Curie agreement number 764759 .

\section{REFERENCES}

[1] U. Betke and M. Henk, Densest lattice packings of 3-polytopes, Comput. Geom. 16 (2000) 157-186. (http://arxiv.org/abs/math/9909172)

[2] H. Cohn, A. Kumar, and G. Minton, Optimal simplices and codes in projective spaces, Geom. Topol. 20 (2016), 1289-1357. (https://arxiv.org/abs/1308.3188)

[3] U. Dieter, How to calculate shortest vectors in a lattice, Math. Comp. 29 (1975), 827-833. (https://www.jstor.org/stable/2005293)

[4] M. Dostert, Geometric Packings of Non-Spherical Shapes, PhD Thesis, University of Cologne, 2017. (http://kups.ub.uni-koeln.de/7706/)

[5] M. Dostert, C. Guzmán, F.M. de Oliveira Filho, and F. Vallentin, New upper bounds for the density of translative packings of three-dimensional convex bodies with tetrahedral symmetry, Discrete Comput. Geom. 58 (2017), 449-481. (https://arxiv.org/abs/1510.02331)

[6] P.M. Gruber, Convex and Discrete Geometry, Springer, Berlin, 2007.

[7] P.M. Gruber and C.G. Lekkerkerker, Geometry of Numbers, North-Holland, Amsterdam, 1987.

[8] O. Hanner, On the uniform convexity of $L^{p}$ and $l^{p}$, Ark. Mat. 3 (1956), 239-244. (https: //doi.org/10.1007/BF02589410).

[9] U.-U. Haus, Gitterpackungen konvexer Korper, Diploma Thesis, Technical University of Berlin, 1999. (https://www.zib.de/groetschel/students/diplomhaus.ps)

[10] E. Jones, E. Oliphant, P. Peterson, et al., SciPy: Open Source Scientific Tools for Python, 2001-. http://www.scipy.org/

[11] Y. Jiao, F.H. Stillinger, and S. Torquato, Optimal packings of superballs, Phys. Rev. E 79 (2009), 041309, 12 pp. (http://arxiv.org/abs/0902.1504)

[12] Y. Jiao, F.H. Stillinger, and S. Torquato, Erratum: Optimal packings of superballs [Phys. Rev. E 79, 041309 (2009)], Phys. Rev. E 84 (2011), 069902.

[13] H. Minkowski, Dichteste gitterförmige Lagerung kongruenter Körper, Nachr. Akad. Wiss. Göttingen Math.-Phys. Kl. II (1904) 311-355. (http://www.digizeitschriften.de/dms/ resolveppn/?PID=GDZPPN002500132)

[14] R. Ni, A.P. Gantapara, J. de Graaf, R. van Roij, and M. Dijkstra, Phase diagram of colloidal hard superballs: from cubes via spheres to octahedra, Soft Matter 8 (2012), 8826-8834. (http: //arxiv.org/abs/1111.4357)

[15] The Sage Development Team, SageMath, the Sage Mathematics Software System (Version 8.2), http://www.sagemath.org 2018. 
Appendix A. Source Code for Proof of Theorem 4.2

We used the following program written in Sage in the proof of Theorem 4.2

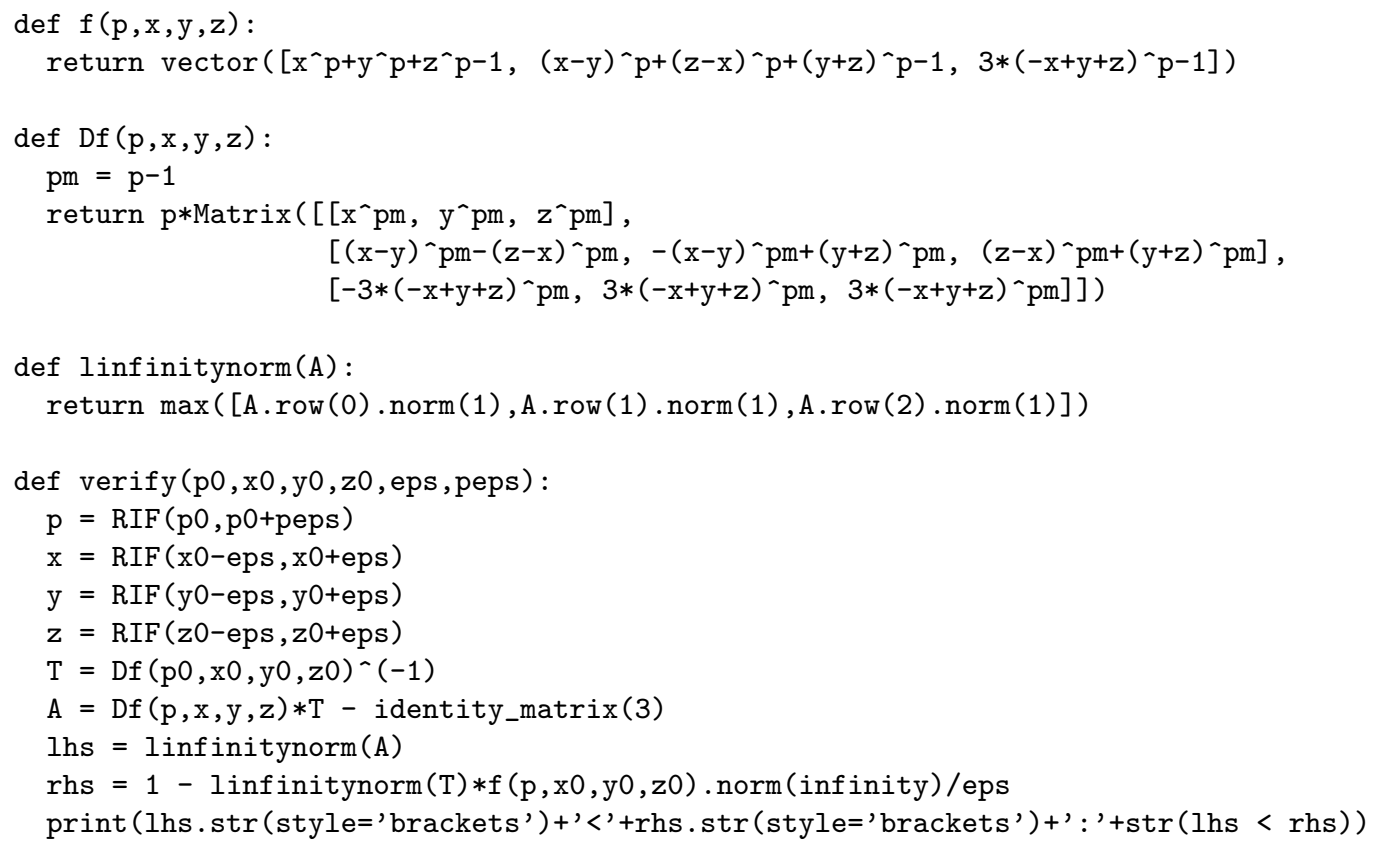

Appendix B. Examples of choices made in the proof of Theorem 4.2

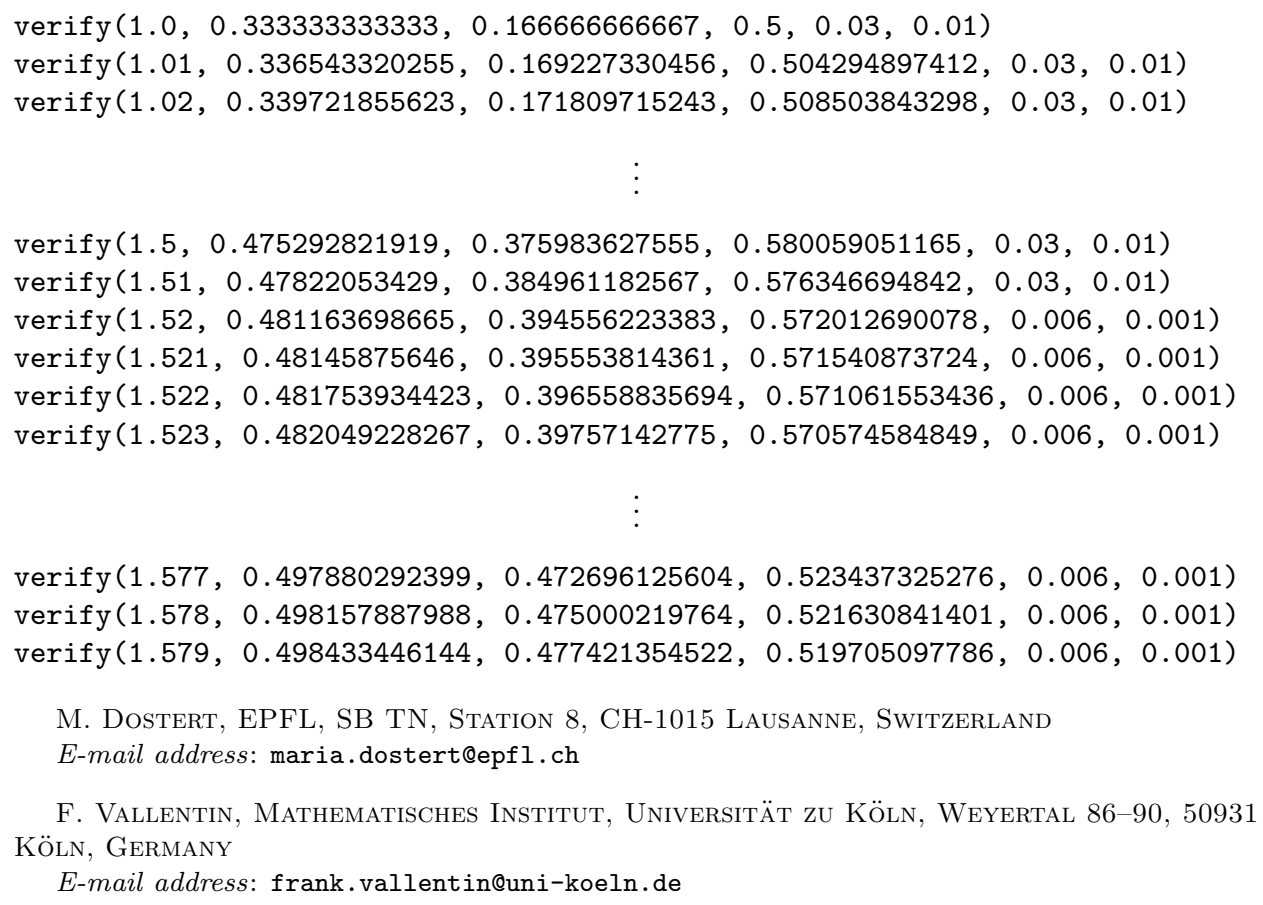

